\title{
The roles of microRNA families in hepatic fibrosis
}

\author{
Xue-Ping Jiang ${ }^{1 \dagger}$, Wen-Bing $\mathrm{Ai}^{2+}$, Lin-Yan Wan ${ }^{1,3}$, Yan-Qiong Zhang ${ }^{1 *}$ and Jiang-Feng Wu ${ }^{1 *}$
}

\begin{abstract}
When hepatocytes are damaged severely, a variety of signaling pathways will be triggered by inflammatory factors and cytokines involving in the process of hepatic fibrosis. The microRNA (miRNA) family consists of several miRNAs which have the potential for synergistic regulation of these signaling pathways. However, it is poor to understand the roles of miRNA family as a whole in hepatic fibrosis. Increasing studies have suggested several miRNA families are related with activation of hepatic stellate cells and hepatic fibrosis through cooperatively regulating certain signaling pathways. During the process of hepatic fibrosis, miR-29 family primarily induces cell apoptosis by modulating phosphatidylinositol 3-kinase/AKT signaling pathway and regulates extracellular matrix accumulation. miR-34 family promotes the progression of hepatic fibrosis by inducing activation of hepatic stellate cells, while miR-378 family suppresses the process in Glis dependent manner. miR-15 family mainly promotes cell proliferation and induces apoptosis. The miR-199 family and miR-200 family are responsible for extracellular matrix deposition and the release of pro-fibrotic cytokines. These miRNA family members play pro-fibrotic or anti-fibrotic roles by targeting genes collectively or respectively which involve in hepatic fibrosis related signaling pathways and hepatic stellate cell activation. Thus, good understandings of molecular mechanisms which are based on miRNA families may provide new ideas for the molecular targeted therapy of hepatic fibrosis in the future.
\end{abstract}

Keywords: microRNA family, Hepatic fibrosis, Hepatic stellate cell, Extracellular matrix, Signaling pathway

\section{Background}

Hepatic fibrosis is the inevitable pathological process of many chronic liver diseases, for instance, non-alcoholic fatty liver disease (NAFLD), non-alcoholic steatohepatitis (NASH), viral hepatitis, sclerosing cholangitis [1]. Once these chronic diseases aggravate further, hepatic fibrosis may progress to liver cirrhosis, even hepatocellular carcinoma (HCC). In pathological condition, hepatic fibrosis is characterized by the imbalance between deposition and degradation of extracellular matrix (ECM) [2]. Activated hepatic stellate cells (HSCs), as the main ECMproducing cells, play a vital role in progression of hepatic fibrosis $[2,3]$.

\footnotetext{
*Correspondence: zhangyanqiong@ctgu.edu.cn; jiangfengwu2011@163.com

${ }^{\dagger}$ Xue-Ping Jiang and Wen-Bing Ai contributed equally to this work ${ }^{1}$ Institute of Organ Fibrosis and Targeted Drug Delivery, China Three Gorges University, 8 Daxue Road, Xiling District, Yichang 443002, China Full list of author information is available at the end of the article
}

The mechanism of hepatic fibrosis is extremely complex covering large numbers of cellular and molecular events [4]. Under the stimulation of exogenous factors, quiescent HSCs (qHSCs) activated, then transformed into myofibroblasts, and secreted mounting ECM, which eventually leads to hepatic fibrosis. Some studies have indicated that the initiation and progress of hepatic fibrosis are related to integrated signaling networks, including MAPK signaling pathway, Wnt signaling pathway, phosphatidylinositol 3-kinase (PI3K)/AKT signaling pathway, Hedgehog (Hh)/Gli signaling pathway, etc. [5]. Therefore, researchers tried their best to find ways to intervene with the key molecules in signaling pathways, and they have found it's possible to reverse or prevent the progression of hepatic fibrosis. For example, IFN- $\gamma$ inhibits TGF- $\beta$-induced phosphorylation of Smad3 and induces the expression of Smad7. Insulin-like growth factor 1 (IGF-1) is able to induce apoptosis and attenuate fibrogenesis. Curcumin may induce the expression of endogenous peroxisome proliferator-activated receptor 
$\gamma$ (PPAR $\gamma$ ) gene and lead to the downregulation of TGF$\beta$. L-Cysteine suppresses HSC proliferation [6]. But the outcomes aren't ideal. As a complex organism, the effects of endogenous regulation on biological processes can't be ignored. Recent studies have highlighted that lots of endogenous factors regulate gene expression. Among them, the studies about miRNAs were more extensive. miRNAs are a class of small, endogenous non-coding RNAs, about 18-25 nucleotides [2]. Mounting evidences have shown that miRNAs involve in lots of complex biological processes, such as cell proliferation, differentiation, apoptosis, and carcinogenesis. miRNAs bind to the $3^{\prime}$-untranlated regions ( $3^{\prime} \mathrm{UTR}$ ) of the target mRNAs. Eventually, the transcription of target genes is inhibited or the stability of the target genes is reduced. Thus, miRNA-mediated RNA interference, as a new mechanism for regulating gene transcription level, is attracting the attention of multitudinous researchers.

In the process of biological evolution and development, several miRNAs form a miRNA family which has sequence homology because of the strong similarity in the sequences of miRNAs. The synergistic effects of the miRNA family, whether globally or individually, have been linked with the HSC activation and the progression of hepatic fibrosis through binding to several signaling pathways related molecules. Thus, in the review, we are going to analyze the internal logic between miRNA families and the signaling pathways and look forward to providing new ideas for the molecular targeted therapy of hepatic fibrosis in the future by pro-fibrotic or antifibrotic roles of miRNA families.

\section{The miRNA-29 family members induce apoptosis of HSCs and reduce the accumulation of ECM}

The miR-29 family has four members, including miR29a, miR-29b1, miR-29b2 and miR-29c [7]. This family is divided into two clusters, miR-29a/b1 which is located on chromosome 7 and miR-29b2/c which is located on chromosome 1 . These family members have the same seed sequence AGCACCA. miR-29a and miR-29c contain 22 nucleotides, which are differ from the only one nucleotide. miR-29b1 and miR-29b2 have the same sequence, and the difference is merely that their genes are located on different chromosomes [8] (Fig. 1). Numerous studies have shown plenty of transcriptional factor binding sites exist in the proximal region of miR-29b1/a and miR29b2/c cluster promoters, such as a Gli binding site, three NF- $\mathrm{kB}$ binding sites, a Smad3 binding site, a CCAAT/ enhancer-binding protein (CEBP) binding site, two T-cell factor/LEF (TCF/LEF) binding sites [9].

Roderburg et al. detected the expression of miR-29b in different hepatic cell compartments isolated from livers of C57BL/ 6 mice by qPCR, it has been proved that

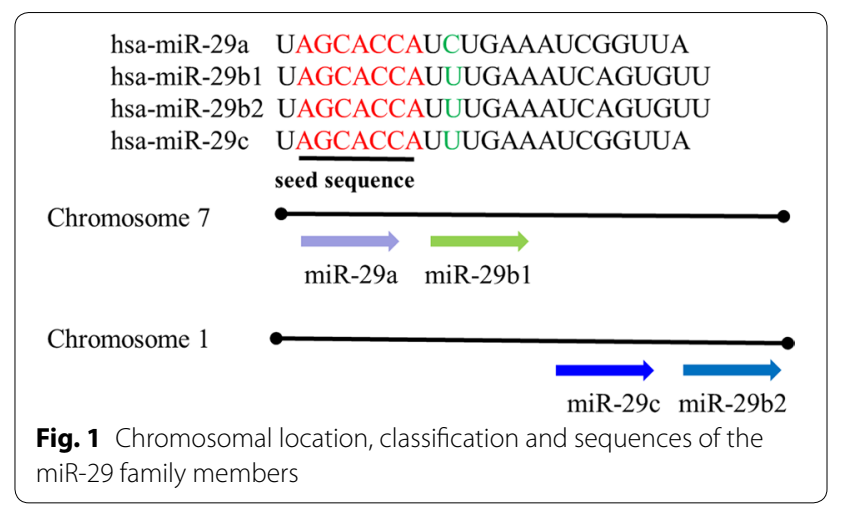

miR-29b shows the highest expression in HSCs, follow by Kupffer cells, hepatocytes, liver sinusoidal endothelial cells (LSEC) [10]. Strong anti-fibrotic effects of miR29 family have been confirmed in liver, kidney, and other organs. The miR-29 family showed dramatic decrease in the two models of hepatic fibrosis compared with nonfibrotic livers as well as in patients with hepatic fibrosis [10]. Moreover, it has been proved that the decrease of miR-29b1/a in hepatocytes also contributes to fibrosis, genetic knockdown of miR-29b1/a has an effect on regulation of genes which involved in fibrosis and enhances susceptibility to fibrosis following a fibrogenic stimulus [11].

Previous studies have proved miR-29 family is related to several signaling pathways in the progression of hepatic fibrosis, such as NF- $\mathrm{KB}$ signaling pathway, $\mathrm{Hh}$ signaling pathway, TGF- $\beta$ signaling pathway, PI3K/AKT signaling pathway, and so on. When NF-kB signaling is damaged, the Hh signaling leads to the activation of HSCs, which promotes fibrogenesis by decreasing the effects of miR-29 on hepatic fibrosis [12]. The crosstalk between miR-29b and TGF- $\beta / \mathrm{Smad} 3$ signaling exists in activated HSCs [13]. There is a Smad response element (TGTCAGTCT) which locates in a highly conserved region $\sim 22 \mathrm{~kb}$ upstream of miR-29b2 promoter. It has been confirmed that Smad3 interacts with the miR-29b2 promoter by chromatin immunoprecipitation (ChIP) assay. The expression of miR-29b is negatively regulated by Smad3 [13]. And miR-29b directly suppresses TGF- $\beta /$ Smad3 signaling which promotes hepatic fibrosis [13].

The activation of PI3K/AKT signaling pathway partly relies on a mass of growth factors, such as platelet derived growth factor $\mathrm{C}$ (PDGF-C) and IGF-1. PDGF$\mathrm{C}$ and IGF-1 can activate these intracellular signaling pathways, however, miR-29a targets PDGF-C and IGF-1 directly [7]. AKT serves as a downstream signaling molecule for activated PI3K, and it triggers a variety of signaling pathways by multiple downstream signaling molecules, such as cyclin D1 (CD-1), P21 and Caspase9. 
miR-29b also regulates negatively the PI3K/AKT signaling pathway by binding to $3^{\prime} \mathrm{UTR}$ of PI3KR1 and AKT3 [14]. Overexpressed miR-29b in activated HSCs leads cell cycle arrest in $\mathrm{G} 1$ phase by the up-regulation of $\mathrm{P} 21$, and then P21 binds and inhibits CD-1. Furthermore, overexpressed miR-29b leads to cellular disassembly and HSCs apoptosis by activating Caspase 9 and triggering the proteolytic cleavage of the PARP [14]. Additionally, miR-29b is related to the formation of ECM [15]. miR-29b targets collagen I $\alpha 1$ (COLI $\alpha 1)$ directly, which is the important component of ECM. And it also targets heat shock protein 47 (HSP47) and lysyl oxidase (LOX), both of which are essential for ECM maturation [16].

Overexpressed miR-29a suppresses the activity of histone deacetylase 4 (HDAC4) which is a member of HDAC class II [17]. The inhibition of class II HDAC activity reduces HSC activation [18]. These findings demonstrate that miR-29a hampers the activation of HSCs (Fig. 2).

miR-29c takes part in the progress of HCC by binding 3'UTR of sirtuin 1 (SIRT1) and promotes liver tumorigenesis [19]. However, no finding has proved it is related with hepatic fibrosis.

\section{The promotion role of $\mathrm{miR}-34$ family in the process of hepatic fibrosis}

The miR-34 family has three members, including miR$34 \mathrm{a}, \mathrm{miR}-34 \mathrm{~b}$ and miR-34c. In mammal, miR-34a localizes on chromosome 1p36. Meanwhile, miR-34b and miR-34c form a cluster which is located on chromosome
$11 \mathrm{q} 23$ and are of the same primary transcript $[20,21]$ (Fig. 3). In mouse, miR-34a is widely expressed with the highest level in brain, while $\mathrm{miR}-34 \mathrm{~b} / \mathrm{c}$ shows high levels in the lung. In human beings, miR-34a is ubiquitously expressed with high levels in the ovary, prostate and testes and low levels in liver and adipose (http://mirnamap.mbc.nctu.edu.tw) [21]. It has demonstrated that the increased expression level of miR-34 associates with downregulation of $\mathrm{NOCH} 1$ protein in liver tissue of rats which treated with tamoxifen [22]. miR-34a was obviously overexpressed after long-term alcohol exposure [23]. The expression of miR-34 family also reflects p53 status and has the potential to regulate both cell cycle arrest and apoptosis [24]. miR-34 family, as a pleiotropic miRNA family, has attracted more and more attention for its cell proliferation, differentiation and apoptosis.

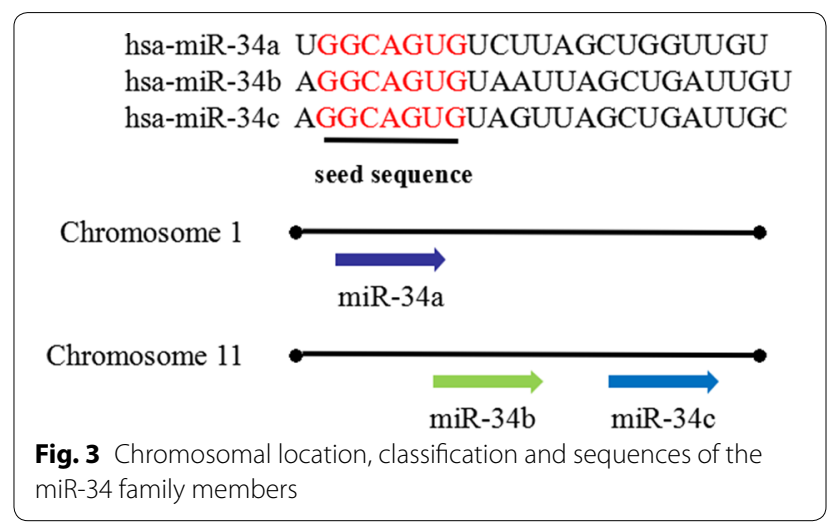

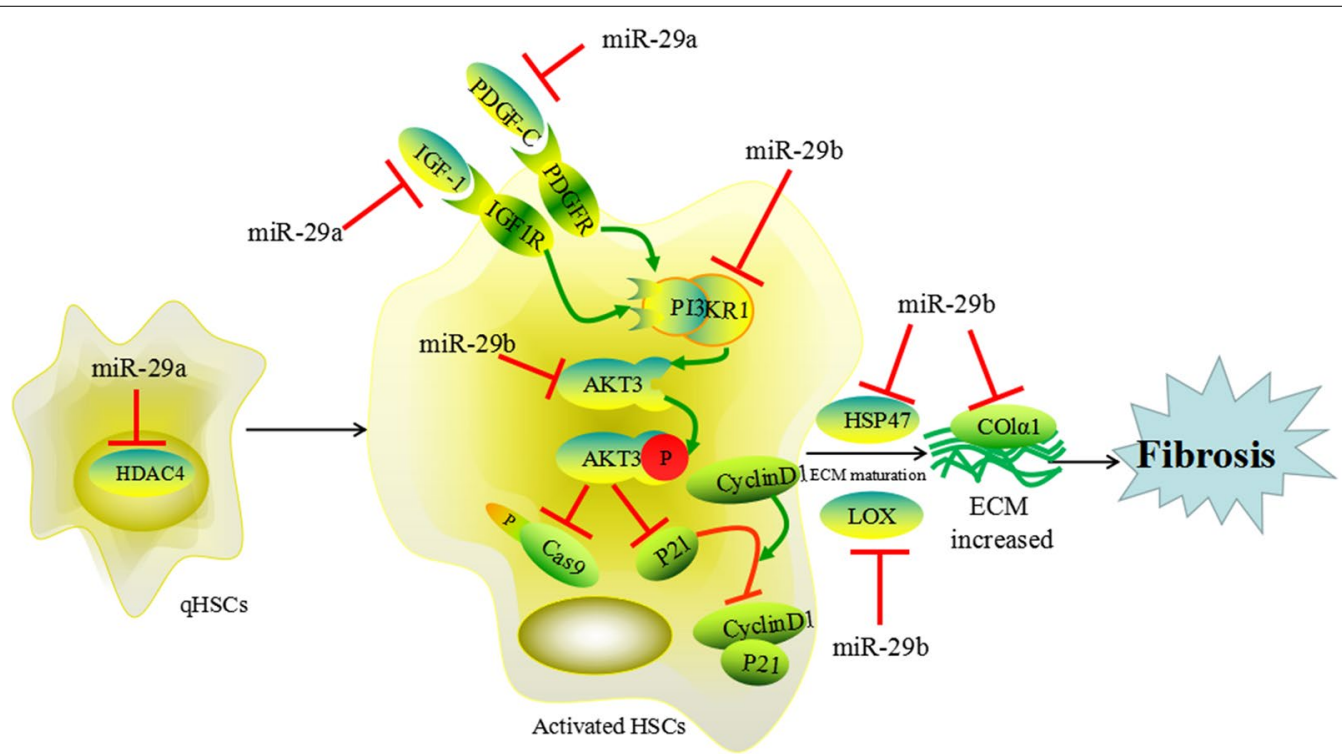

Fig. 2 The miR-29 family members regulate the progress of hepatic fibrosis by following ways: (1) miR-29a targets HDAC4 to inhibit the activation of qHSCs; (2) miR-29b targets AKT3 and PI3KR1, then induces cell apoptosis; (3) miR-29a inhibits PDGF-C and IGF-1 to suppress PI3K/AKT signaling pathway; (4) miR-29b inhibits ECM maturation and ECM accumulation by targeting HSP47, LOX and COLla1, respectively 
In rats, miR-34a promotes the activation of HSCs through targeting acyl-CoA synthetase long-chain family member 1 (ACSL1) which plays an important role in the storage pathway of lipid metabolism in liver [25]. The silence of miR-34a upregulates expression level of ACSL1 resulting in promoting lipogenesis, and then the accumulation of fatty acids in HSCs inhibits the activation of HSCs. The accumulation of vitamin A-containing lipid droplets is characteristic of qHSCs [26]. Furthermore, miR-34a increases the deposition of ECM proteins in rats, such as COLI, desmin and $\alpha$-SMA, by interaction with 3'UTR of ACSL1 [27].

It has been proved that PPAR $\gamma$, a nuclear transcription factor, is a direct target of miR-34a and miR-34c through luciferase reporter assays in HSCs [28]. Overexpressed miR-34a and miR-34c decrease the expression of PPAR $\gamma$ and increase the level of $\alpha$-SMA. PPAR $\gamma$, an anti-fibrotic factor, contributes to maintaining the quiescent phenotype of HSCs [28]. In hepatocytes, miR-34a directly targets caspase2 (CASP2) and SIRT1. During the process of alcoholic liver injury, SIRT1 which partially localizes in the cytoplasm leads to increase sensitivity to apoptosis [23]. CASP2 activates mitochondrial apoptosis pathway resulting in cell apoptosis ultimately [29]. Overexpression of the miR-34a also upregulates the expressions of matrix metalloproteinase 2 and 9 (MMP2 and MMP9) in alcoholic liver diseases. It has been proved that the MMPs are vital parts in cell remodeling and tissue repair during fibrogenic process [23]. Taken together, these findings have proved that miR-34a and miR-34c play promotion roles in process of hepatic fibrosis caused by chronic liver injury, likely abnormal lipid metabolism and alcohol injury (Fig. 4).

\section{The miR-15 family members have a positive impact on TGF- $\beta$ signaling pathway and a negative impact on PI3K/AKT signaling pathway}

The miR-15 family consists of six highly conserved miRNAs, including miR-15a/b, miR-16, miR-195, miR-497 and miR-322 [30]. They share the same seed sequence AGCAGC (Fig. 5). There are two miR-15/16 clusters in mammals. In normal tissues, miR-15a and miR-16-1 are expressed highly as a miRNA cluster from an intron region of the deleted in lymphocytic leukemia 2 (DLEU2, a non-coding transcript that affects cell proliferation) transcript. Several binding sites, c-Myc, c-Myb, or PPAR, exist in the DLEU2 promoter region. The expression of miR15a/16-1 cluster is controlled positively by c-Myb and PPAR and it is also controlled negatively by c-Myc, thus regulating DLEU2 transcription [31]. The miR-15b/16-2 cluster is hosted by structural maintenance of chromosome protein 4 (SMC4) genes which is an evolutionarily-conserved ATPase and associates with

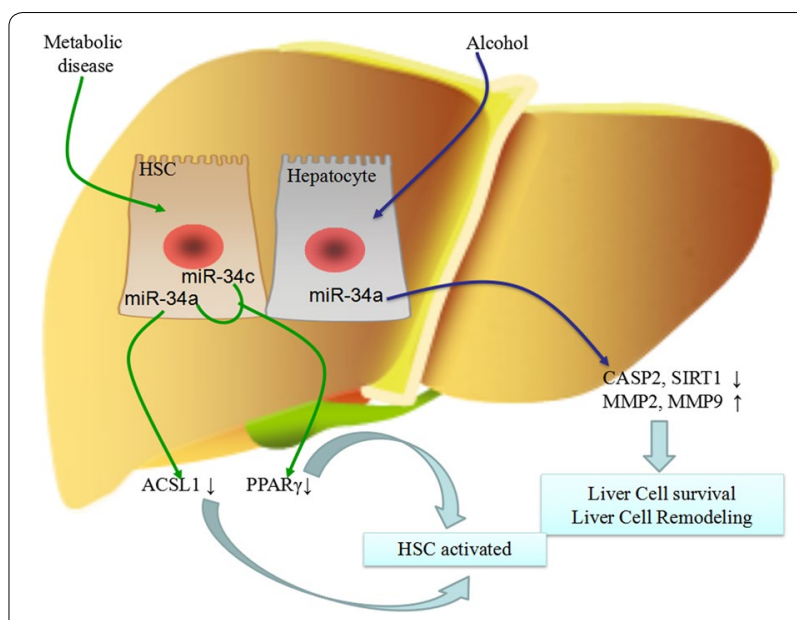

Fig. 4 The miR-34 family members regulate hepatic fibrosis which is induced by chronic liver injury via following ways: (1) miR-34a targets ACSL1 and PPARY to promote the activation of HSCs; (2) miR-34c targets PPARy to change the phenotype of HSCs; (3) in hepatocyte, miR-34a inhibits apotosis by downregulating CASP2 and SIRT1. (4) miR-34a participates in cell remodeling by increasing the level of MMP2 and MMP9

hsa-miR-15a UAGCAGCACAUAAUGGUUUGUG hsa-miR-15b UAGCAGCACAUCAUGGUUUACA hsa-miR-16 UAGCAGCACGUAAAUAUUGGCG hsa-miR-195 UAGCAGCACAGAAAUAUUGGC hsa-miR-497 CAGCAGCAAUUCAUGUUUUGAA hsa-miR-322 CAGCAGCACACUGUGGUUUGU

$$
\text { seed sequence }
$$

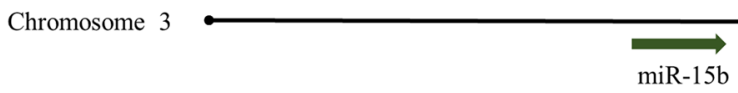

Chromosome 13

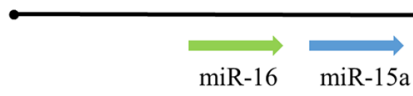

Chromosome 17

$$
\underset{\text { miR-195 }}{\Rightarrow \text { miR-497 }}
$$

Chromosome X

miR-322

Fig. 5 Chromosomal location, classification and sequences of the miR-15 family members

chromosome stability and dynamics [32]. The sequences of miR-15a/16-1 and miR-15b/16-2 are highly conserved. Mature miR-16 is produced from miR-16-1 and miR16-2 [32]. The expression levels of miR-15a, miR-15b and miR-16 were increased in rat livers which perfused with hyperosmotic solution. Upregulated miR-15a, miR-15b and miR-16 contribute to cell death through the inhibition of anti-apoptotic genes, such as $\mathrm{Bcl} 2$, CD-1, protein carboxyl-O-methyltransferase (Pcmt1) [33]. miR-15 
family regulates TGF- $\beta$ signaling pathway by targeting TGFßr1, Smad3, Smad7, p38 and endoglin directly or indirectly in cardiac fibrosis [30].

As reported in previous study, hepatitis B virus (HBV) mRNAs sponge off miR-15a and upregulate Smad7 which is a novel target of miR-15a, then inhibit TGF- $\beta / \mathrm{Smad}$ pathway. It has proved that miR-15a has a positive effect on TGF- $\beta /$ Smad pathway in HBV-infected patients [34]. Hepatitis $\mathrm{C}$ virus (HCV) infection causes chronic hepatitis, which may progress to hepatic fibrosis, even HCC. The HCV patients with hepatic fibrosis express high levels of miR-16 [35, 36]. miR-16 suppresses the expressions of hepatocyte growth factor (HGF) and Smad7 in HCV-induced hepatic fibrosis [36]. HGF, amitogen for hepatocytes, inhibits the progress of hepatic fibrosis via suppressing the expression of TGF- $\beta$ [37]. Moreover, HGF plays an anti-fibrotic role by increasing collagenase expression and promoting the collagen degradation [38]. In TGF- $\beta /$ Smad signaling pathway, TGF- $\beta$ plays a profibrotic role, however, Smad7 plays an anti-fibrotic role and alleviates the extent of hepatic fibrosis [36]. All these evidences mean that the miR-16 is a pro-fibrotic factor and has a positive effect on TGF- $\beta /$ Smad signaling pathway.

The PI3K/AKT pathway, an important signaling cascade, promotes cell proliferation and inhibits apoptosis [39]. Activated AKT modulates downstream factors including B-cell lymphoma-2 (Bcl-2), CD-1, cyclin E1. Guo et al. has proved that the expressions of miR-15b and miR-16 are increased obviously in activated rat HSCs by using microarray analysis. miR-15b and miR-16 target $\mathrm{Bcl}-2$ directly which is known to be a potent anti-apoptosis factor. As a result, the downstream factors of Bcl-2 are activated greatly, such as Caspase3, 8, 9, which reflect the irreversible phase of apoptosis. These studies show miR$15 \mathrm{~b}$ and miR-16 play pro-apoptosis roles in HSCs [26]. The expression of CD-1 is markedly increased in rat culture-activated HSCs, which has an effect on keeping the integrity of the G1/S checkpoint. Overexpressed miR-16 reduces the level of $\mathrm{CD}-1$ by binding 7 complementary nucleotides (TGCTGCT) at posttranscriptional level, then inhibits cell proliferation [40]. Cyclin E1, an important initiation factor of $\mathrm{S}$ phase, promotes HSC proliferation [41, 42]. miR-195 downregulates the expression of cyclin E1 by binding its 3'UTR. It means that miR-195 plays an anti-fibrotic role in hepatic fibrosis by targeting cyclin E1 [42] (Fig. 6).

\section{Regulatory function of miR-200 family on their target genes in the pathogenesis of hepatic fibrosis} The miR-200 family has five members, including miR200a, miR-200b, miR-200c, miR-429 and miR-141. They are divided into two different clusters. One of the

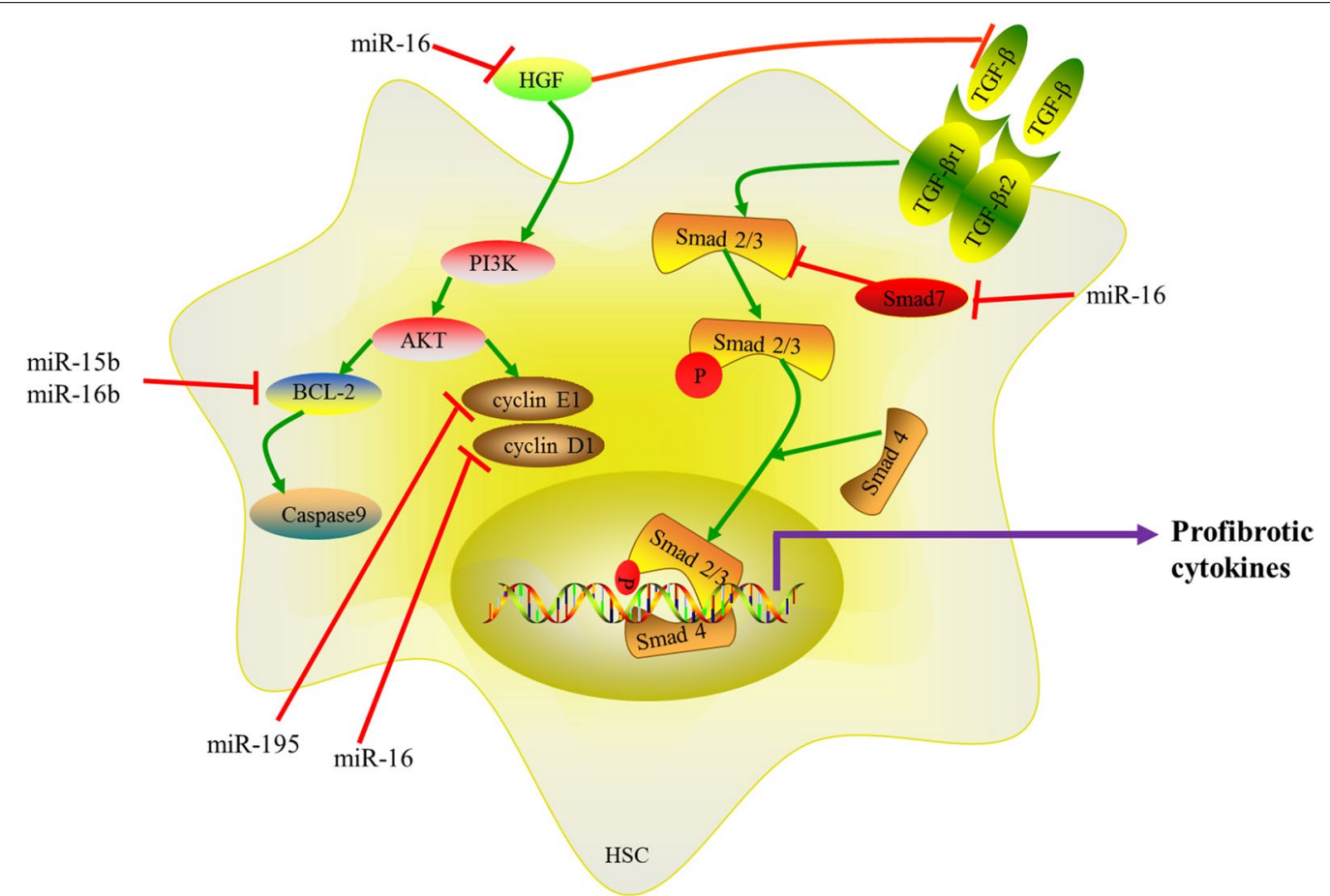

Fig. 6 The miR-15 family members regulate the progress of hepatic fibrosis by following ways: (1) miR-16 targets HGF and Smad7 to promote TGF- $\beta /$ Smad signaling pathway; (2) miR-16 inhibits cell proliferation by targeting CD1; (3) miR-195 downregulates cyclin E1 to inhibit cell proliferation; (4) miR-15b and miR-16b promote apoptosis by targeting Bcl-2 
clusters is located on chromosome 1, which contains miR-200a/b/429 members, the other cluster includes miR$200 \mathrm{c} / 141$, which is located on chromosome 12 . Based on the structure of these members, they are also divided into two functional groups, the difference between them is about the third nucleotide of seed sequences. Functional group 1 is miR-200a/141, whose seed sequence is AACACUG, the other is $\mathrm{miR}-200 \mathrm{~b} / \mathrm{c} / 429$, whose seed sequence is AAUACUG [43] (Fig. 7). The expression of $\mathrm{miR}-200 \mathrm{a} / \mathrm{b} / 429$ cluster is driven by SP1 which is a ubiquitous activating transcription factor [44]. It has been found that miR-200 family inhibits ZEB1 and ZEB2, the E-cadherin transcriptional repressors, to restore an epithelial phenotype in breast cancer cell lines for the first time [45].

Large numbers of evidences have proved that miR-200 family participates in fibrosis. A single injection of premiR-200b inhibits COLI expression level in renal fibrosis [46]. miR-200b inhibits the process of intestinal fibrosis through repressing ZEB1 and ZEB2 [47]. In NASH model, upregulated miR-200b leads to the changed expression of fibrotic-relevant genes, such as, the decrease of ZEB1 and increase of E-cadherin. Nevertheless, transfection of mouse primary hepatocytes with miR-200b upregulated the level of E-cadherin only [48]. Cholestasis is an important cause of hepatic fibrosis. In cholestatic livers, increased miR-200a is inversely related to FOXA2 mRNA expression, decreased FOXA2 might contribute to the activation of IL-6/STAT3 signaling pathway [49]. During development of hepatic fibrosis, Sun and co-workers have proved miR-200a inhibits cell proliferation by inducing G0/G1 phase arrest in TGF- $\beta$ treated HSCs. They also declared that miR-200a may partly regulate TGF- $\beta$ signaling pathway via translational suppression of TGF- $\beta 2$ expression [50]. It has been proved that miR-200a suppresses $\beta$-catenin in the protein level, a key factor of Wnt $/ \beta$-catenin signaling pathway, which participates in liver remodeling and HSC activation [50]. And $\beta$-catenin stimulates the expression of fibrosis-related genes

hsa-miR-141 UAACACUGUCUGGUAAAGAUGG hsa-miR-200a UAACACUGUCUGGUAACGAUGU hsa-miR-200b UAAUACUGCCUGGUAAUGAUGA hsa-miR-200c UAAUACUGCCGGGUAAUGAUGGA hsa-miR-429 UAAUACUGUCUGGUAAAACCGU

$$
\text { seed sequence }
$$

Chromosome 1

$$
\text { miR-200b miR-200a miR-429 }
$$

Chromosome 12

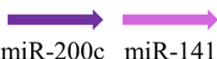

Fig. 7 Chromosomal location, classification and sequences of the miR-200 family members by forming a $\beta$-catenin-LEF-TCF transcriptional complex in nucleus [50, 51].

Overexpressed miR-200a downregulates the expression of Kelch-like ECH-associated protein 1 (Keap1) resulting in upregulation of nuclear factor-erythroid-2-related factor 2 (Nrf2) in HSCs [52]. The exposure of HSCs to reactive oxygen species (ROS) can promote their proliferation and thus promote process of hepatic fibrosis in PI3K dependent manner [1]. When cells subject to ROS, Keap1 is inactivated. Nrf2, as an antioxidant factor, binds antioxidant response element (ARE) in nucleus resulting in the transcriptional activation of many cytoprotective genes which involve in the elimination of ROS in Keap1/ Nrf2 system [53]. When miR-200a mimic was transfected into HSCs, the level of $\alpha$-SMA decreased [52]. HCV is a common cause of hepatic fibrosis, and it has identified that HCV infection increases the level of miR-200c. FAS associated phosphatase 1 (FAP-1) as the direct target of miR-200c, is downregulated in the chronic HCV infected liver. Decreased expression of FAP-1 promotes the activation of proto-oncogene tyrosine-protein kinase $\mathrm{Src}(\mathrm{cSrc})$. Activated cSrc modulates TGF- $\beta$ signaling pathway which promotes the process of hepatic fibrosis by phosphorylating and activating TGF- $\beta$ type II receptor [54] (Fig. 8).

\section{The pro-fibrogenic effects of miR-199 family members}

The miR-199 family has three members, including miR199a1, miR-199a2 and miR-199b. miR-199a1 and miR$199 \mathrm{a} 2$ have the same mature sequence and locate on chromosome 19, chromosome 1 , respectively. Three members differ from only two nucleotides [55] (Fig. 9).

miR-199a, a conserved small non-coding RNA, is identified in inner ear hair cells firstly [56]. Transfection of HK2 cells with miR-199a-3p analog also significantly decreased the expression of SOCS7 and increased the phosphorylation of STAT3 [57]. Previous study has proved STAT3 inhibitors ameliorate renal fibrosis [58]. miR-199a-3p as an pro-fibrotic factor involves in renal fibrosis by SOCS7/STAT3 axis. It suggests that the expression levels of miR-199a-5p and miR-199a-3p are increased in two different models of hepatic fibrosis by using miRNA based platform. And increased miR$199 a-5 p$ is correlated with the severity of hepatic fibrosis $[59,60]$. TGF- $\beta 1$ inhibits the expression of HGF by up-regulating miR-199a-3p. HGF is required for normal tissue repair and it has been demonstrated HGF blocks fibrotic remodeling in the liver [61]. Researchers have found that the miR-199a-5p elevated and the caveolin-1 (CAV1) expression decreased in HSCs treated by TGF- $\beta$ [62]. CAV1, a target of miR-199a-5p, internalizes TGF receptors into caveolae and this internalisation represses TGF- $\beta$ signaling pathway [63]. Moreover, CAV1 inhibits 


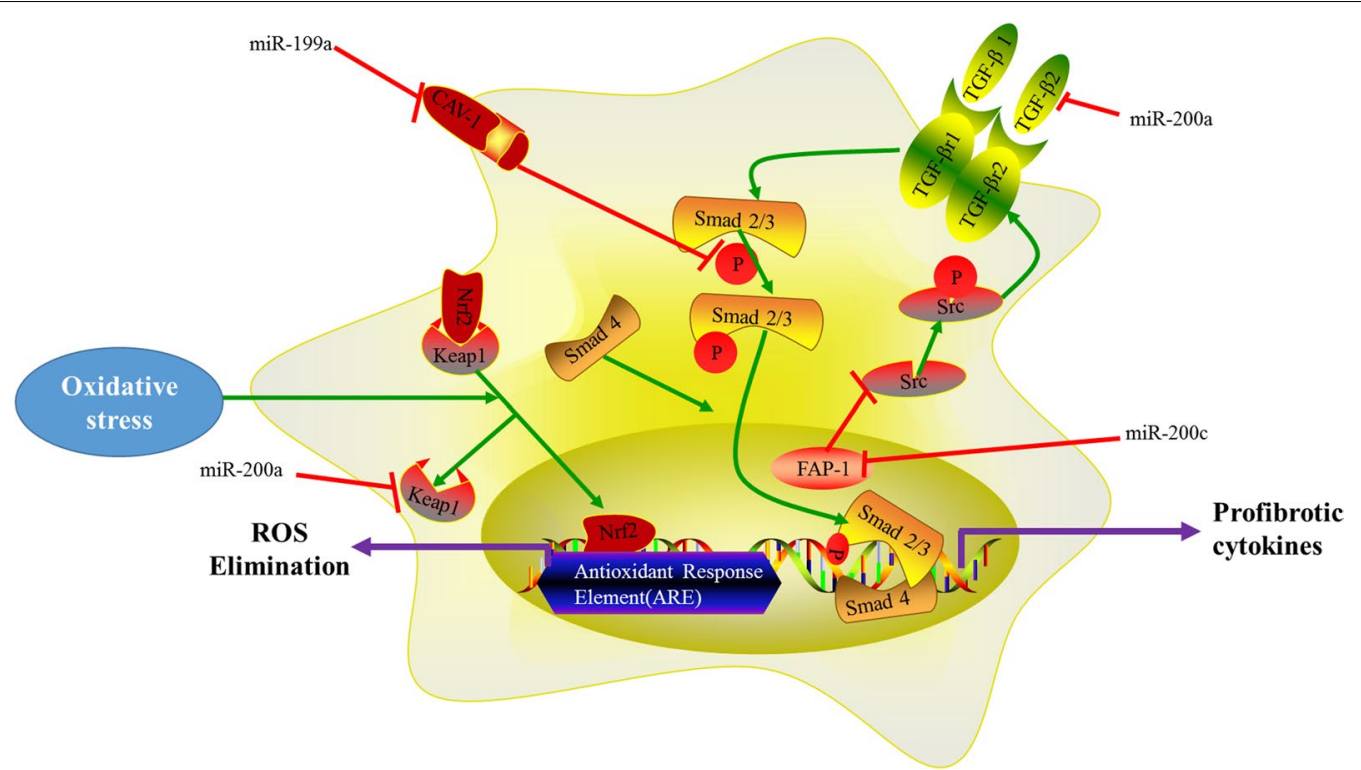

Fig. 8 The miR-199 family and miR-200 family members regulate hepatic fibrosis by following ways: (1) miR-199a reduces the level of CAV-1 and then promotes the phosphorylation of Smad2; (2) miR-200a downregulates Keap1 and activates the transcription of many cytoprotective genes which involve in the elimination of ROS; (3) miR-200a inhibits TGF- $\beta / S$ mad pathway by targeting TGF- $\beta 2$; (4) miR-200c targets FAP1 and then activates $\mathrm{CS}$ rc resulting in promoting TGF- $\beta /$ Smad pathway

TGF- $\beta$ mediated the phosphorylation of Smad2 [64]. Overexpressed miR-199a increases the levels of MMP13, tissue inhibitors of metalloproteinases-1 (TIMP1) and $\alpha 1$ procollagen, all of which involve in the imbalance of ECM degradation and synthesis [60] (Fig. 9).

\section{The miR-378 family members suppress the activation of HSCs}

The miR-378 family includes eleven members, miR-378a, miR-378b, miR-378c, miR-378d1, miR-378d2, miR-378e, miR-378f, miR-378 g, miR-378 h, miR-378i and miR-378j [65]. Among them, miR-378a has two mature strands, miR-378a-3p and miR-378a-5p, which locate on chromosome 5 in humans [65]. They originate from perxisome proliferation activated receptor gamma coactivator $1 \beta$ (PPARGC1 $\beta$ ) gene encoding PGC-1 $\beta$ [66].

It has identified that the expression levels of miR378a-3p, miR-378b and miR-378d are repressed in CCl4treated liver by microarray and real-time quantitative reverse transcriptase PCR analyses. Moreover, miR-378 decreases in liver tissues of rats with dimethylnitrosamine induced hepatic fibrosis [20]. There is a positive correlation between the severity of liver fibrosis and the level of the activated Hh signaling pathway which promotes hepatic fibrosis $[67,68]$. In activated Hh signaling pathway, cytoplasmic Smo promotes the translocation of p65 into the nucleus. Activated p65 inhibits the transcription of miR-378a by binding to the promoter (or

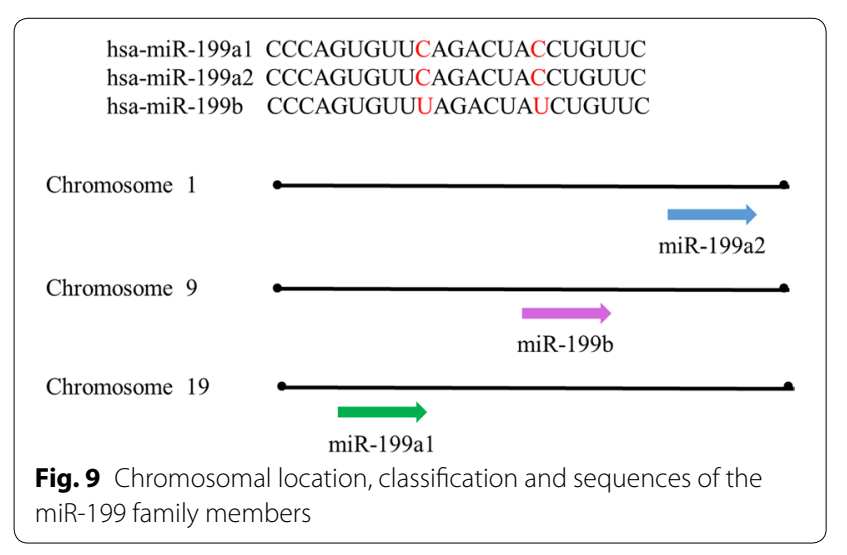

control element) of the miR-378a [68]. In activated HSCs being transfected with miR-378a-3p mimic, the profibrotic gene expressions of Vimentin, $\alpha$-SMA, and CoLI $\alpha 1$ decreased, the level of Gli3 was also downregulated. It has been proved that the miR-378a-3p suppresses activation of HSCs through directly targeting Gli3 [68].

\section{Conclusion}

Despite researches about biological significance and utility of miRNAs in pathological process of hepatic fibrosis are developing rapidly, our understanding about the roles of miRNA families in hepatic fibrosis is so poor. Although some features of miRNA family members-such as their effects on target genes-are well described (Table 1), the 
Table 1 potential miRNAs involved in hepatic fibrosis

\begin{tabular}{lll}
\hline miRNA & $\begin{array}{l}\text { Expression during the } \\
\text { process of hepatic fibrosis }\end{array}$ & Target genes \\
\hline miR-29a & Downregulated & PDGF-C, IGF-1, HDAC4 \\
miR-29b & Downregulated & $\begin{array}{c}\text { PI3KR1, AKT3, COLa1, LOX, } \\
\text { HSP47 }\end{array}$ \\
miR-34a & Upregulated & ACSL1, PPARY, CASP2, SIRT1 \\
miR-34b & Upregulated & PPARY \\
miR-15a & Downregulated & Smad7 \\
miR-15b & Upregulated & Bcl-2 \\
miR-16 & Upregulated & Smad7, HGF, BCl-2, CD-1 \\
miR-195 & Downregulated & Cyclin E1 \\
miR-200a & Downregulated & TGF- 32, Keap1 \\
miR-200c & Upregulated & FAP1 \\
miR-199a & Upregulated & CAV1 \\
miR-378a & Downregulated & Gli3 \\
\hline
\end{tabular}

cell-specific regulatory potential of miRNA family as a whole in the liver awaits further investigation. Specially, several questions need to be solved.

Firstly, does the miRNA family have more significant effects on hepatic fibrosis than a single miRNA? Secondly, are there any interactions among the miRNA family members? Finally, from a clinical perspective, can miRNA families act as valuable diagnostic and prognostic biomarkers? Despite the difficulties will exist for a long time, it's worth for us investigating the important roles of miRNA families in hepatic fibrosis and exploring some ways to treat hepatic fibrosis. Perhaps in the future, upregulating or downregulating the expression of miRNA families may be efficient and more specific approaches for therapy of liver fibrosis.

\begin{abstract}
Abbreviations
miRNA: microRNA; PI3K: phosphatidylinositol 3-kinase; HSC: hepatic stellate cell; ECM: extracellular matrix; NAFLD: non-alcoholic fatty liver disease; NASH: non-alcoholic steatohepatitis; HCC: hepatocellular carcinoma; qHSC: quiescent hepatic stellate cell; Hh: Hedgehog; PPARY: peroxisome proliferator-activated receptor $\gamma ; 3^{\prime} U T R$ : 3'-untranlated regions; CEBP: CCAAT/enhancer-binding protein; TCF/LEF: T cell factor/LEF; LSEC: liver sinusoidal endothelial cells; ChIP: chromatin immunoprecipitation; PDGF-C: platelet derived growth factor C; IGF-1: insulin-like growth factor 1; CD-1: cyclin D1; COLla1: collagen la1; HSP47: heat shock protein 47; LOX: lysyl oxidase; HDAC4: histone deacetylase 4; ACSL1: acyl-COA synthetase long-chain family member 1; CASP2: caspase2; SIRT1: sirtuin 1; MMPs: matrix metalloproteinases; DLEU2: deleted in lymphocytic leukemia 2; SMC4: structural maintenance of chromosome protein 4; Pcmt1: protein carboxyl-O-methyltransferase; HBV: hepatitis B virus; HCV: hepatitis C virus; HGF: hepatocyte growth factor; BCl-2: B-cell lymphoma-2; Keap 1: Kelch-like ECH-associated protein 1; Nrf2: nuclear factor-erythroid2-related factor 2; ROS: reactive oxygen species; ARE: antioxidant response element; FAP-1: FAS associated phosphatase 1; cSrc: proto-oncogene tyrosineprotein kinase Src; TIMP1: tissue inhibitors of metalloproteinases-1; PPARGC1 $\beta$ : perxisome proliferation activated receptor gamma coactivator $1 \beta$.
\end{abstract}

\section{Authors' contributions}

XPJ and WBA contributed equally to this work and wrote the manuscript. LYW gave some helps for this work. YQZ and JFW revised and approved the article prior to its being submitted for publication. All authors read and approved the final manuscript.

\section{Author details}

${ }^{1}$ Institute of Organ Fibrosis and Targeted Drug Delivery, China Three Gorges University, 8 Daxue Road, Xiling District, Yichang 443002, China. ${ }^{2}$ The Yiling Hospital of Yichang, 31 Donghu Road, Yi Ling District, Yichang 443100, Hubei, China. ${ }^{3}$ The RenMin Hospital, China Three Gorges University, 31 Huti Subdistrict, Xi Ling District, Yichang 443000, Hubei, China.

\section{Acknowledgements}

The authors thank Shanbing Yin for English language editing. We are thankful for the financial support of the National Natural Science Foundation of China (Grant Numbers: 81670555, 81200307).

\section{Competing interests}

The authors declare that they have no competing interests.

\section{Consent for publication}

All authors reviewed the manuscript and consent for publication.

\section{Funding}

The National Natural Science Foundation of China (Grant Numbers: 81670555 , 81200307) has given financial support.

\section{Publisher's Note}

Springer Nature remains neutral with regard to jurisdictional claims in published maps and institutional affiliations.

Received: 12 April 2017 Accepted: 26 June 2017

Published online: 04 July 2017

\section{References}

1. Lambrecht J, et al. The role of miRNAs in stress-responsive hepatic stellate cells during liver fibrosis. Front Physiol. 2015;6:209.

2. Jiang $X$, et al. MicroRNAs and the regulation of fibrosis. FEBS J. 2010:277:2015-21.

3. Teng KY, et al. Role of noncoding RNAs as biomarker and therapeutic targets for liver fibrosis. Gene Expr. 2015;16:155-62.

4. Toosi AE. Liver fibrosis: causes and methods of assessment. Rom J Intern Med. 2015;53:304-14.

5. Yang JJ, et al. Non-coding RNA-mediated epigenetic regulation of liver fibrosis. Metabolism. 2015;64:1386-94.

6. Ahmad R, et al. Understanding the mechanism of hepatic fibrosis and potential therapeutic approaches. Saudi J Gastroenterol. 2012;18:155.

7. Kwiecinski $\mathrm{M}$, et al. Expression of platelet-derived growth factor- $\mathrm{C}$ and insulin-like growth factor I in hepatic stellate cells is inhibited by miR-29. Lab Invest. 2012;92:978-87.

8. Noetel A, et al. microRNA are central players in anti- and profibrotic gene regulation during liver fibrosis. Front Physiol. 2012;3:49.

9. Kriegel AJ, et al. The miR-29 family: genomics, cell biology, and relevance to renal and cardiovascular injury. Physiol Genomics. 2012;44:237-44.

10. Roderburg C, et al. Micro-RNA profiling reveals a role for miR-29 in human and murine liver fibrosis. Hepatology. 2011;53:209-18.

11. Kogure T, et al. Hepatic miR-29ab1 expression modulates chronic hepatic injury. J Cell Mol Med. 2012;16:2647-54.

12. Hyun J, et al. Potential role of Hedgehog signaling and microRNA-29 in liver fibrosis of IKKß-deficient mouse. J Mol Histol. 2014;45:103-12.

13. Liang $C$, et al. Suppressive effect of microRNA-29b on hepatic stellate cell activation and its crosstalk with TGF- $\beta 1 /$ Smad3. Cell Biochem Funct. 2016:34:326-33.

14. Wang J, et al. microRNA-29b prevents liver fibrosis by attenuating hepatic stellate cell activation and inducing apoptosis through targeting PI3K/ AKT pathway. Oncotarget. 2015;6:7325-38.

15. Ogawa T, et al. Suppression of type I collagen production by microRNA29b in cultured human stellate cells. Biochem Biophys Res Commun. 2010;391:316-21. 
16. Zhang Y, et al. MiR-29b inhibits collagen maturation in hepatic stellate cells through down-regulating the expression of HSP47 and lysyl oxidase. Biochem Biophys Res Commun. 2014;446:940-4.

17. Huang $\mathrm{YH}$, et al. Activation of Mir-29a in activated hepatic stellate cells modulates its profibrogenic phenotype through inhibition of histone deacetylases 4. PLoS ONE. 2015;10:e0136453.

18. Mannaerts I, et al. Class II HDAC inhibition hampers hepatic stellate cell activation by induction of microRNA-29. PLOS ONE. 2013;8:e55786.

19. Bae HJ, et al. MicroRNA-29c functions as a tumor suppressor by direct targeting oncogenic SIRT1 in hepatocellular carcinoma. Oncogene. 2014:33:2557-67.

20. Li WQ, et al. The rno-miR-34 family is upregulated and targets ACSL1 in dimethylnitrosamine-induced hepatic fibrosis in rats. FEBS J. 2011;278:1522-32.

21. Agostini $M$, et al. miR-34: from bench to bedside. Oncotarget. 2014;5:872-81.

22. Pogribny IP, et al. Induction of microRNAome deregulation in rat liver by long-term tamoxifen exposure. Mutat Res. 2007;619:30-7.

23. Meng $F$, et al. Epigenetic regulation of miR-34a expression in alcoholic liver injury. Am J Pathol. 2012;181:804-17.

24. He L, et al. A microRNA component of the p53 tumour suppressor network. Nature. 2007:447:1130-4.

25. Parkes HA. Overexpression of acyl-CoA synthetase-1 increases lipid deposition in hepatic (HepG2) cells and rodent liver in vivo. Am J Physiol Endocrinol Metab. 2006;291:E737-44.

26. Guo CJ, et al. miR-15b and miR-16 are implicated in activation of the rat hepatic stellate cell: an essential role for apoptosis. J Hepatol. 2009;50:766-78.

27. Yan $\mathrm{G}$, et al. MicroRNA-34a promotes hepatic stellate cell activation via targeting ACSL1. Med Sci Monit. 2015;21:3008-15.

28. Li $\mathrm{X}$, et al. microRNA-34a and microRNA-34c promote the activation of human hepatic stellate cells by targeting peroxisome proliferator-activated receptor $\gamma$. Mol Med Rep. 2015;11:1017-24.

29. Tang L, et al. MiR-125a-5p decreases after long non-coding RNA HOTAIR knockdown to promote cancer cell apoptosis by releasing caspase 2. Cell Death Dis. 2016;7:e2137.

30. Tijsen AJ, et al. The microRNA-15 family inhibits the TGF $\beta$-pathway in the heart. Cardiovasc Res. 2014;104:61-71.

31. Zheng ZM, et al. Regulation of cellular miRNA expression by human papillomaviruses. Biochim Biophys Acta. 2011;1809:668-77.

32. Yue J, et al. Conservation of miR-15a/16-1 and miR-15b/16-2 clusters, Mamm Genome. 2010;21:88-94

33. Santosa D, et al. Hyperosmotic stress activates the expression of members of the miR-15/107 family and induces downregulation of antiapoptotic genes in rat liver. Sci Rep. 2015;5:12292.

34. Liu N, et al. Hepatitis B virus regulates apoptosis and tumorigenesis through the microRNA-15a-Smad7-transforming growth factor beta pathway. J Virol. 2015;89:2739-49.

35. Fognani $E$, et al. Role of microRNA profile modifications in hepatitis $C$ virus-related mixed cryoglobulinemia. PLOS ONE. 2013;8:e62965.

36. Zhu B, et al. Increased miR-16 expression induced by hepatitis $C$ virus infection promotes liver fibrosis through downregulation of hepatocyte growth factor and Smad7. Arch Virol. 2015;160:2043-50.

37. Jiang Z, et al. Attenuation of hepatic fibrosis through ultrasoundmicrobubble-mediated HGF gene transfer in rats. Clin Imaging. 2013;37:104-10.

38. Kim WH, et al. Growth inhibition and apoptosis in liver myofibroblasts promoted by hepatocyte growth factor leads to resolution from liver cirrhosis. Am J Pathol. 2005;166:1017-28.

39. Fulda S. Modulation of mitochondrial apoptosis by PI3K inhibitors. Mitochondrion. 2013;13:195-8.

40. Guo CJ, et al. Effects of upregulated expression of microRNA-16 on biological properties of culture-activated hepatic stellate cells. Apoptosis. 2009:14:1331-40

41. Golias $\mathrm{CH}$, et al. Cell proliferation and cell cycle control: a mini review. Int Clin Pract. 2004;58:1134-41.

42. Sekiya $Y$, et al. Down-regulation of cyclin E1 expression by microRNA-195 accounts for interferon- $\beta$-induced inhibition of hepatic stellate cell proliferation. J Cell Physiol. 2011;226:2535-42.
43. Senfter $D$, et al. The microRNA-200 family: still much to discover. Biomol Concepts. 2016;7:311-9.

44. Kolesnikoff N, et al. Specificity protein 1 (Sp1) maintains basal epithelial expression of the miR-200 family: implications for epithelial-mesenchymal transition. J Biol Chem. 2014;289:11194-205.

45. Chung AC, et al. microRNAs in renal fibrosis. Front Physiol. 2015;6:50.

46. Oba S, et al. miR-200b precursor can ameliorate renal tubulointerstitial fibrosis. PLOS ONE. 2010;5:e13614.

47. Chen Y, et al. miR-200b is involved in intestinal fibrosis of Crohn's disease. Int J Mol Med. 2012;29:601-6.

48. Pogribny IP, et al. Difference in expression of hepatic microRNAs miR29c, miR-34a, miR-155, and miR-200b is associated with strain-specific susceptibility to dietary nonalcoholic steatohepatitis in mice. Lab Invest. 2010;90:1437-46

49. Xiao Y, et al. Dysregulated miR-124 and miR-200 expression contribute to cholangiocyte proliferation in the cholestatic liver by targeting IL-6/STAT3 signalling. J Hepatol. 2015;62:889-96.

50. Sun $X$, et al. Participation of miR-200a in TGF- $\beta 1$-mediated hepatic stellate cell activation. Mol Cell Biochem. 2014;388:11-23.

51. Cheng JH, et al. Wnt antagonism inhibits hepatic stellate cell activation and liver fibrosis. Am J Physiol Gastrointest Liver Physiol. 2008:294:G39-49.

52. Yang JJ, et al. MicroRNA-200a controls Nrf2 activation by target Keap1 in hepatic stellate cell proliferation and fibrosis. Cell Signal. 2014;26:2381-9.

53. Mitsuishi Y, et al. The Keap1-Nrf2 system in cancers: stress response and anabolic metabolism. Front Oncol. 2012;2:200.

54. Ramachandran S, et al. Hepatitis C virus induced miR200c down modulates FAP-1, a negative regulator of Src signaling and promotes hepatic fibrosis. PLOS ONE. 2013:8:e70744.

55. Li Z, et al. miR-199-sponge transgenic mice develop physiological cardiac hypertrophy. Cardiovasc Res. 2016;110:258-67.

56. Friedman LM, et al. MicroRNAs are essential for development and function of inner ear hair cells in vertebrates. Proc Natl Acad Sci USA. 2009;106:7915-20.

57. Yang R, et al. p53 induces miR199a-3p to suppress SOCS7 for STAT3 activation and renal fibrosis in UUO. Sci Rep. 2017;7:43409.

58. Pang $M$, et al. A novel STAT3 inhibitor, S3I-201, attenuates renal interstitial fibroblast activation and interstitial fibrosis in obstructive nephropathy. Kidney Int. 2010;78:257-68.

59. Roy $\mathrm{S}$, et al. The role of miRNAs in the regulation of inflammatory processes during hepatofibrogenesis. Hepatobiliary Surg Nutr. 2015;4:24-33.

60. Murakami Y, et al. The progression of liver fibrosis is related with overexpression of the miR-199 and 200 families. PLOS ONE. 2011;6:e16081.

61. Mungunsukh $\mathrm{O}$, et al. Transforming growth factor- $\beta 1$ selectively inhibits hepatocyte growth factor expression via a micro-RNA-199-dependent posttranscriptional mechanism. Mol Biol Cell. 2013:24:2088-97.

62. Lino Cardenas CL, et al. miR-199a-5p Is upregulated during fibrogenic response to tissue injury and mediates TGFbeta-induced lung fibroblast activation by targeting caveolin-1. PLoS Genet. 2013;9:e1003291.

63. O'Reilly S. MicroRNAs in fibrosis: opportunities and challenges. Arthritis Res Ther. 2016:18:11.

64. Razani B, et al. Caveolin-1 regulates transforming growth factor (TGF)beta/SMAD signaling through an interaction with the TGF-beta type receptor. J Biol Chem. 2001;276:6727-38.

65. Megiorni F, et al. Deep Sequencing the microRNA profile in rhabdomyosarcoma reveals down-regulation of miR-378 family members. BMC Cancer. 2014:14:880.

66. Bellafante $\mathrm{E}$, et al. Hepatic-specific activation of peroxisome proliferatoractivated receptor $\gamma$ coactivator- $1 \beta$ protects against steatohepatitis. Hepatology. 2013;57:1343-56.

67. Choi SS, et al. The role of Hedgehog signaling in fibrogenic liver repair. Int J Biochem Cell Biol. 2011:43:238-44.

68. Hyun J, et al. MicroRNA-378 limits activation of hepatic stellate cells and liver fibrosis by suppressing Gli3 expression. Nat Commun. 2016;7:10993. 